\title{
Circuit Theory Based Analysis of CMUT Arrays with Very Large Number of Cells
}

\author{
H.Kağan Oğuz, Abdullah Atalar, and Hayrettin Köymen \\ Bilkent University, Electrical and Electronics Engineering Department, Ankara, Turkey \\ Email: oguz@ee.bilkent.edu.tr
}

\begin{abstract}
We have recently developed a circuit theory based method to analyze large CMUT arrays and shown that mutual acoustic interactions significantly influence the transducer performance. We connect each cell in the array to a radiation impedance matrix that contains the mutual radiation impedance between every pair of cells, in addition to their self radiation impedances. However, efficient analysis of very large arrays is challenging, which may become computationally cumbersome. To partition the problem, we electrically drive a single element in the array and keep the rest undriven but biased and with their electrical ports terminated with a load. The radiation impedance matrix can be partitioned and rearranged to represent these loads in a reduced form. In this way, only the driven element can be simulated by coupling its cells through this reduced impedance matrix. This method considerably reduces the number of cells and the size of the original radiation impedance matrix at the expense of calculating the inverse of a large complex symmetric matrix.
\end{abstract}

\section{INTRODUCTION}

Many applications require capacitive micromachined ultrasonic transducers (CMUTs) to be used in the form of large arrays to attain a better performance. It is known that mutual acoustic interactions between the cells have significant effects on array performance [1], [2] and recently different methods are used to study them [3]-[5]. To benefit from its full potential, it is necessary to use analysis tools that are capable of handling a large number of CMUT cells. In this regard, finite element analysis (FEA) tools are unfit for use, because of the size and complexity of the resulting problem.

In our recent work [5], we investigated the effects of mutual acoustic interactions between the cells of CMUT array elements and showed that the performance of the array is significanlty influenced by this phenomenon. The acoustic terminals of each cell are coupled through a radiation impedance matrix, where the self and mutual radiation impedances are included. It is crucial to couple every pairs of cells in the array, otherwise the solution becomes inaccurate. In this way, we obtained a practical way of analyzing the performance of large arrays using the circuit theory principles. If the radiation impedance matrix becomes very large, under some circumstances we can partition the problem to reduce its size.

\section{Reduced RAdiation Impedance MAtrix}

In CMUT arrays, the acoustic force at the radiation interface of each cell can be interpreted in matrix form as in Fig. 1, where $F_{i}$ and $U_{i}$ represent the rms force and $r m s$ velocity of the individual cells, respectively. The square matrix is the radiation impedance matrix, $\mathbf{Z}$, where the diagonal elements are the self and off-diagonals are the mutual radiation impedances.

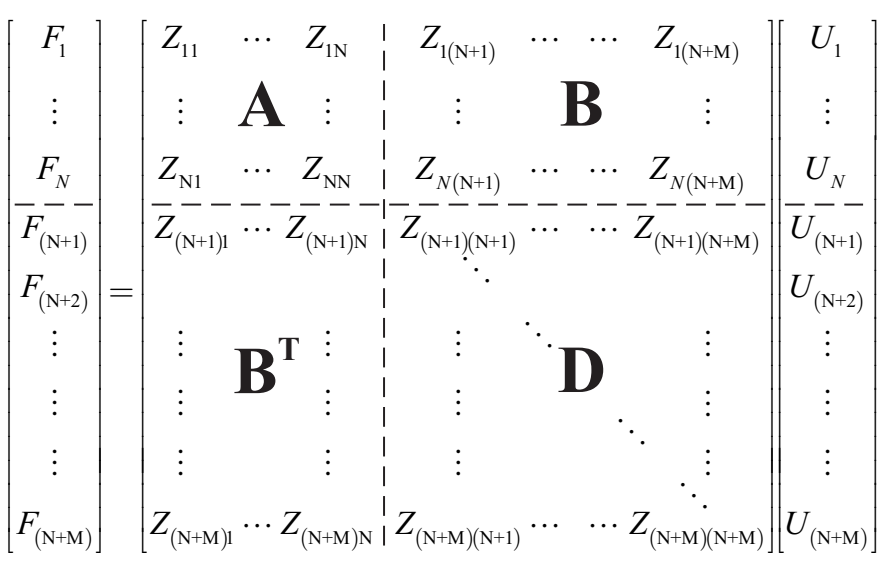

Fig. 1. Radiation impedance matrix, $\mathbf{Z}$, is depicted, where the equivalent circuit variables $F_{i}$ and $U_{i}$ represent the force and rms velocity of the individual cells in a CMUT array. $\mathbf{Z}$ is partitioned such that $N$ and $M$ are the number of cells in the driven and undriven elements, respectively.

A typical CMUT array contains hundreds of cells in each of its elements, which may end up with over 100,000 cells in the entire array. For such a large array, the computation of the radiation impedance matrix and the solution of the circuit theory based model require an excessive time [5]. Ultrasonic arrays are usually actuated by applying voltage pulses to groups of elements located side to side in the array. In this case, we may partition $\mathbf{Z}$ as shown in Fig. 1, where $N$ is the number of cells in the electrically driven elements and $N+M(M \gg N)$ is the total number of cells in the array. The circuit variables of the operating elements are aggregated at the upper left corner of $\mathbf{Z}$, which is denoted as $\mathbf{A}$. Note that if the rest of the elements were constrained to be stationary (i.e. $\left.U_{(N+1)}, U_{(N+2)}, \cdots U_{(N+M)}=0\right)$, then only $\mathbf{A}$ would determine the overall response, because it is the radiation impedance matrix of the operating elements when they are located on an infinite rigid plane baffle. On the other hand, in the small signal regime, we have the following relation for the cells existing in the undriven elements

$$
\frac{F_{(N+i)}}{U_{(N+i)}}=-Z_{e m} \text { for } i=1,2, \cdots M,
$$

where $Z_{\text {em }}$ is the electromechanical impedance of each cell 
observed at its radiation interface.

To reduce the size of the problem without any loss of accuracy, we may electrically drive a small number of elements in the array and keep the rest undriven (but biased or unbiased), with their electrical ports terminated with a load. Then, by using (1), $\mathbf{Z}$ can be rearranged to represent these loads in a reduced form as

$$
\left[F_{1} \cdots F_{N}\right]^{T}=\left[\mathbf{A}-\mathbf{B}(\mathbf{E}+\mathbf{D})^{-1} \mathbf{B}^{T}\right]\left[U_{1} \cdots U_{N}\right]^{T}
$$

where $\mathbf{B}$ and $\mathbf{D}$ are the remaining partitions of $\mathbf{Z}$ as shown in Fig. 1 and $\mathbf{E}=-Z_{e m} \mathbf{I}_{M}$, where $\mathbf{I}_{M}$ is the identity matrix. In this way, only the driven elements can be simulated by coupling its cells through this reduced impedance matrix. This method considerably reduces the number of cells and the size of the original radiation impedance matrix at the expense of calculating the inverse of the complex symmetric matrix $(\mathbf{E}+\mathbf{D})$. Finally, if needed, the separately calculated responses of the elements can be added using superposition principle to find the total response.

For CMUTs, $Z_{e m}$ depends on the electrical termination load and/or the DC bias voltage applied on the undriven elements. Fig. 2 depicts the small signal equivalent circuit of a single CMUT cell when it is unbiased and biased with its electrical port terminated with a load, $Z_{L}$. Derivation of this circuit model is described in detail in [6].

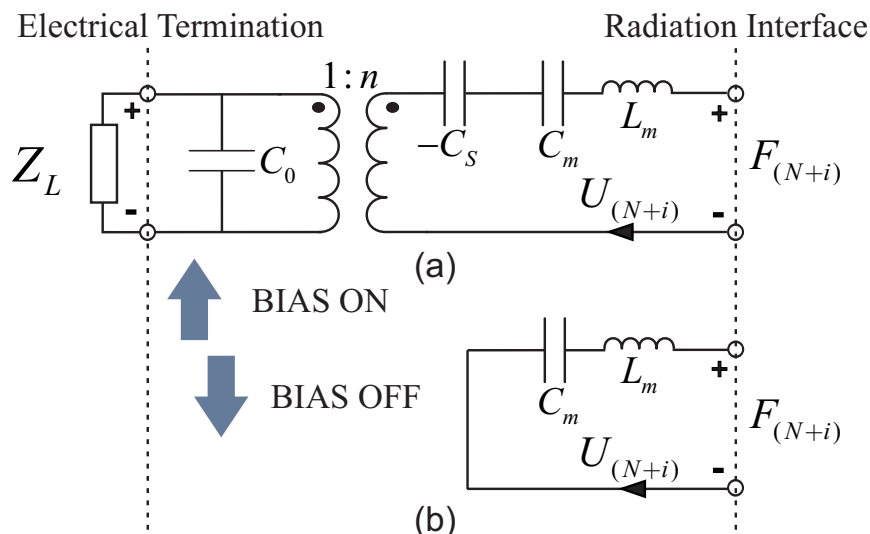

Fig. 2. The small signal equivalent circuit model of (a) DC biased and (b) unbiased single CMUT cell.

\section{ELEMENTS IN LARGE ARRAYS WITH UNDRIVEN NEIGHBORS}

In [7] and [5], we studied the effects of mutual acoustic interactions for different sized elements of 1-D CMUT arrays. In this section, the frequency response of one of those elements is reanalyzed when there are undriven neighbor elements. The CMUT cell dimensions and material properties used in the simulations are listed in Table I.

We analyzed arrays, which have 3, 5 and 7 elements, and examined their performance in water depending on the surroundings of one of its driven elements. Fig. 3 depicts the 7 element array, where each element with 88 cells is nearly $10 \lambda$ long and $\lambda / 2$ wide at $3.5 \mathrm{MHz}$ in water. Each time only
TABLE I

PROPERTIES OF THE CMUT CELL USED IN THE SIMULATIONS

\begin{tabular}{ll||ll}
\hline Dimensions & & Material Properties & \\
\hline Membrane radius & $53 \mu \mathrm{m}$ & Young's modulus & $320 \mathrm{GPa}$ \\
Membrane thickness & $4.3 \mu \mathrm{m}$ & Density & $3.27 \mathrm{~g} / \mathrm{cm}^{3}$ \\
Gap height & $200 \mathrm{~nm}$ & Poisson's ratio & 0.263 \\
Insulator thickness & $100 \mathrm{~nm}$ & Insulator permittivity & 4 \\
\hline
\end{tabular}

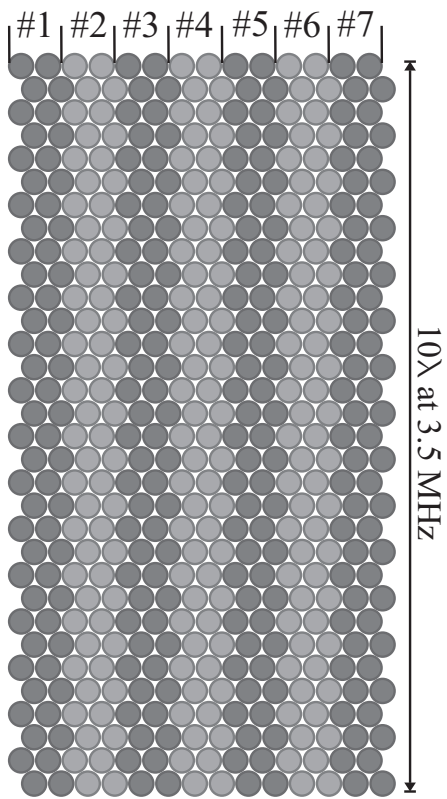

Fig. 3. 7 side to side CMUT array elements which are nearly $10 \lambda$ long at $3.5 \mathrm{MHz}$ in water.

a single element is excited and it is loaded with the reduced radiation impedance matrix to represent the loads due to the undriven elements.

First, all of the elements are DC biased at $70 \%$ of the collapse voltage $(68 \mathrm{~V})$. Fig. 4(a) shows the electrical conductance when only the element located in the middle (\#4) of the array is driven with $1 \mathrm{~V}$ peak $\mathrm{AC}$ voltage and there exists one, two and three undriven elements at each side of it. The electrical ports of the undriven elements are loaded with a high impedance electrical load $\left(Z_{L} \simeq \infty\right)$. Fig. 4(b) plots the response of the 7 element array when the driven element is shifted to element \#3, \#2 and \#1, respectively. In each graph, the values for a single element placed on an infinite rigid baffle are also given for reference. These results show that a single element in a rigid baffle has many spurious resonances, where one of them, around $3.75 \mathrm{MHz}$, overrides the others. As the number of undriven elements and their positions change the number and rate of these spurious resonances also change. This suggest that assuming nearby undriven elements as rigid boundaries is not correct because the membranes have low mechanical impedance.

We also considered $Z_{L}=50 \Omega$ termination at the electrical port of the undriven elements, which yielded almost the same results as for $Z_{L} \simeq \infty$. This is because the reactance of the clamped capacitance is already too low compared to the rest 


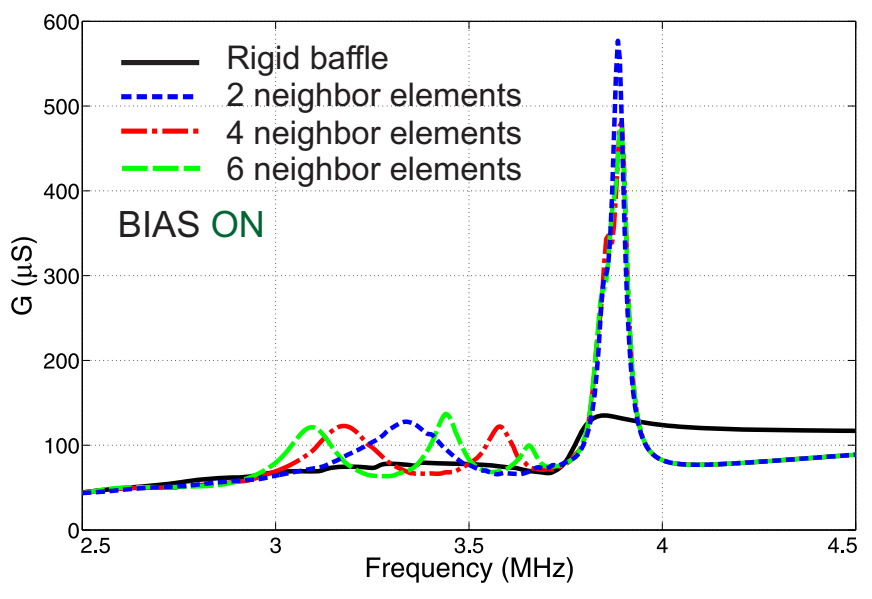

(a)

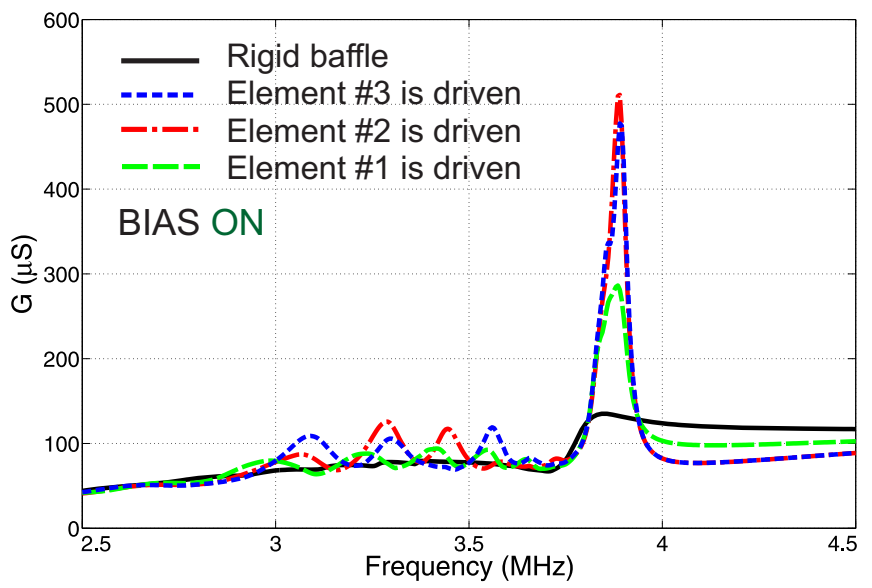

(b)

Fig. 4. Electrical conductances of a single $10 \lambda$ long CMUT array element. The variations show the effects of mutual interactions when the undriven elements are biased and their electrical ports are left open. (a) The middle element is driven when there are 1,2 , and 3 undriven elements at each side. (b) Driven element is shifted from center to side in a 7 element array.

of the series (mechanical) reactance.

On the other hand, when the undriven elements are not DC biased an electromechanical transduction does not occur within them. In this case, the electrical capacitance $\left(C_{0}\right)$, the spring softening capacitance $\left(-C_{S}\right)$ and the electrical load $\left(Z_{L}\right)$ of the undriven cells are not coupled to the mechanical side. Only the compliance $\left(C_{m}\right)$ and the mass $\left(L_{m}\right)$ of the membranes of these elements contribute to the overall radiation impedance experienced by the driven element, as shown in Fig. 2(b). The analysis made above is repeated to carry this case into effect. The results are plotted in Fig. 5. Once again the spurious resonances are highly influenced by the new boundary conditions due to this termination.

\section{CONCLUSION}

We presented a circuit theory based method to analyze the performance of large CMUT arrays by partitioning the radiation impedance matrix when there are undriven elements in the array. The process involves an inversion of a large

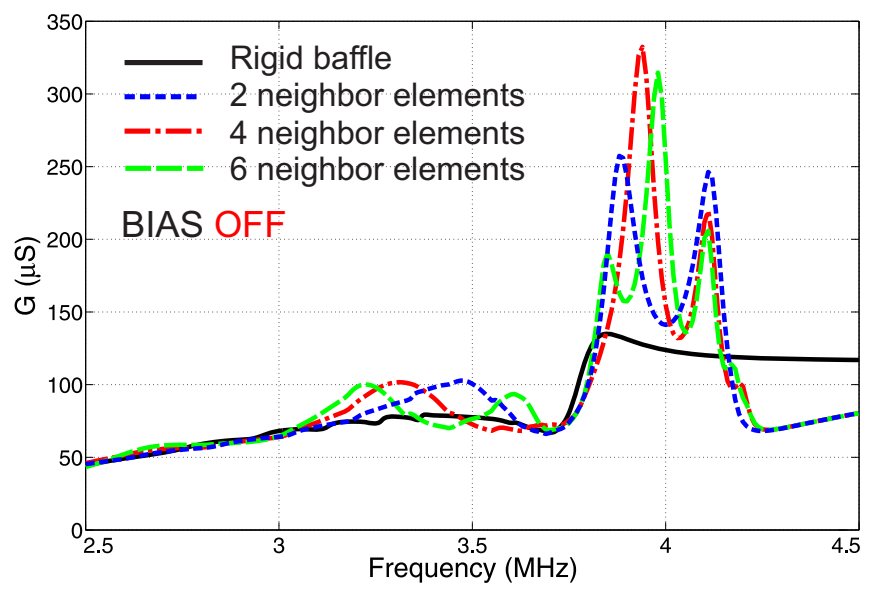

(a)

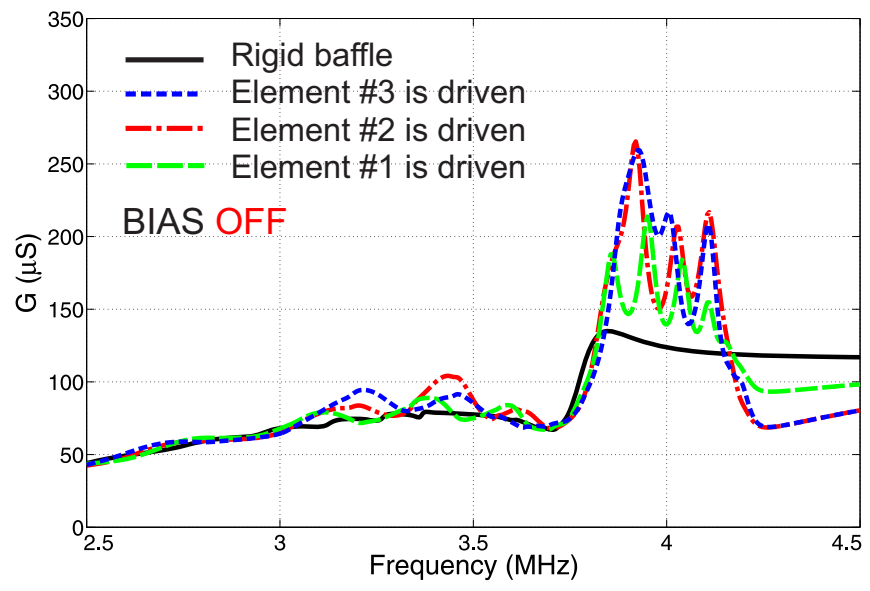

(b)

Fig. 5. Electrical conductances of a single $10 \lambda$ long CMUT array element. The variations show the effects of mutual interactions when the undriven elements are not biased. (a) The middle element is driven when there are 1, 2, and 3 undriven elements at each side. (b) Driven element is shifted from center to side in a 7 element array.

matrix. In the small signal regime, the method is very accurate and it is practical for analyzing the frequency response.

The low mechanical impedance of CMUT arrays makes them suitable for immersion applications, but they also become susceptible to mutual acoustic interactions and many spurious resonances occur. Introduction of loss in the electrical side does not have a significant effect. Coating layers may alter these resonances but they are not helpful to reduce their amplitudes unless they are very lossy. However, hard protective coating layers can be effective, since it decreases the mechanical compliance of the membranes.

The mutual effects are less effective on arrays with low membrane radius to thickness ratios. It is shown in [5] that for a ratio of 8 , the mutual effects are insignificant.

In practice, arrays are not operated in rigid baffle. We believe that arrays located on baffles other than rigid can cause a loss mechanism to reduce these spurious resonances. Presently, we are considering the possibility of employing 
the presented method to convert the rigid baffle into any impedance baffle.

\section{ACKNOWLEDGMENT}

This work was supported by the Scientific and Technological Research Council of Turkey (TUBITAK) under project grant 110E216. A. Atalar acknowledges the support of the Turkish Academy of Sciences (TUBA).

\section{REFERENCES}

[1] K. Niederer, P. C. Eccardt, H. Meixner, and R. Lerch, "Micromachined transducer design for minimized generation of surface waves," in Proc. IEEE Ultrasonics Symp., vol. 2, 1999, pp. 1137-1139 vol.2.

[2] A. Caronti, A. Savoia, G. Caliano, and M. Pappalardo, "Acoustic coupling in capacitive microfabricated ultrasonic transducers: modeling and experiments." IEEE Trans. Ultrason. Ferroelectr. Freq. Control, vol. 52, no. 12 , pp. 2220-2234, 2005.

[3] C. Meynier, F. Teston, and D. Certon, "A multiscale model for array of capacitive micromachined ultrasonic transducers." J. Acoust. Soc. Am. vol. 128, no. 5, pp. 2549-61, 2010.

[4] K. K. Park and B. T. Khuri-Yakub, "Dynamic response of an array of flexural plates in acoustic medium," The Journal of the Acoustical Society of America, vol. 132, no. 4, pp. 2292-2303, 2012.

[5] H. K. Oğuz, A. Atalar, and H. Köymen, "Equivalent Circuit-Based Analysis of CMUT Cell Dynamics in Arrays." IEEE Trans. Ultrason. Ferroelectr. Freq. Control, vol. 60, no. 5, pp. 1016-1024, 2013.

[6] H. Köymen, A. Atalar, E. Aydoğdu, C. Kocabaş, H. K. Oğuz, S. Olcum, A. Ozgurluk, and A. Ünlügedik, "An improved lumped element nonlinear circuit model for a circular CMUT cell," IEEE Trans. Ultrason. Ferroelectr. Freq. Control, vol. 59, no. 8, pp. 1791-99, 2012.

[7] H. K. Oğuz, A. Atalar, and H. Köymen, "Analysis of Mutual Acoustic Coupling in CMUT Arrays Using an Accurate Lumped Element Nonlinear Equivalent Circuit Model," in Proc. IEEE Ultrasonics Symp., 2012. 